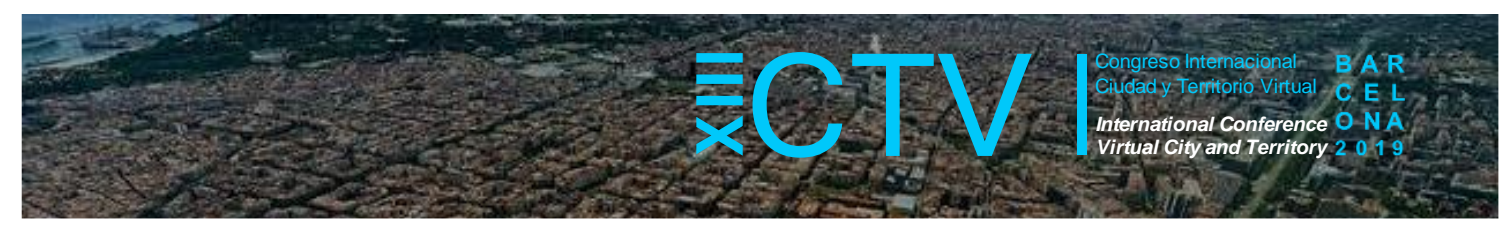

\title{
LAS MEDIDAS DE INTEGRACIÓN COMO INSTRUMENTO REGENERADOR Y DE INVERSIÓN EN LA CIUDAD
}

\author{
Sicilia Pedroza, Lorena ${ }^{1 *}$; Gómez Peltier, Gustavo ${ }^{2}$
}

Remisión inicial: 2019-05-21; Remisión definitiva: 2019-09-20; Publicación: 2019-12-21

Citación: Sicilia, L. y Gómez, G. (2019). Las medidas de integración como instrumento regenerador y de inversión en la ciudad. En XIII CTV 2019 Proceedings: XIII International Conference on Virtual City and Territory: "Challenges and paradigms of the contemporary city": UPC, Barcelona, October 2-4, 2019. Barcelona: CPSV, 2019, p. 8416. E-ISSN 2604-6512. DOI http://dx.doi.org/10.5821/ctv.8416

\section{Resumen}

Las teorías que fundamentan la idea de ciudad parten de la base de un consenso racional supeditado a la existencia de sujetos con los mismos derechos representados por gobiernos democráticos y eficientes. Sin embargo, en los países subdesarrollados la base conceptual sobre la cual se construye la idea de ciudad difiere de la establecida en países con estructuras democráticas y prácticas sociales más desarrolladas.

El impacto del desarrollo inmobiliario generado por la producción capitalista de las ciudades en América Latina no solo ha acelerado y profundizado los procesos de segregación territorial y los conflictos sociales, sino que también han sobrepasado la capacidad de planear, financiar y gestionar el desarrollo urbano de los gobiernos locales y nacionales. La acelerada dinámica del desarrollo inmobiliario también ha trastocado la significación ciudadana sobre la ciudad y sus relaciones, sin que tampoco se haya podido construir una nueva idea de ciudad que integre y ordene eficazmente esta dinámica y no solo la rechace.

En la Ciudad de México encontramos uno de los mejores ejemplos de estos procesos de transformación urbana en el que resulta muy claro que los instrumentos de planeación y gestión de la ciudad están rebasados, pero también presenta una oportunidad incomparable de replantear estos instrumentos ante la implementación de un nuevo marco jurídico y reglamentario derivado de la implementación de la primera Constitución Política de la Ciudad de México. En este caso de estudio, se explica en forma general la problemática existente y se presenta una propuesta metodológica a partir de la cual la administración de la ciudad cuente con mejores herramientas para realizar una evaluación sistémica de los impactos urbanos para nuevos proyectos, así como un ejemplo práctico de cómo pueden mitigar y compensar sus efectos e incluso como un instrumento de financiamiento del desarrollo urbano a escala barrial.

Esta propuesta parte de un caso -actualmente en desarrollo- que realiza el Tecnológico de Monterrey (TEC) en su Campus Ciudad de México, el cual tuvo importantes daños como consecuencia del sismo del 19 de septiembre de 2019 lo que también generó un conjunto de oportunidades para replantear el papel de sus instalaciones y operación con relación a su entorno urbano no solo desde una perspectiva de corresponsabilidad con su entorno urbano y social, sino también como un factor de cambio y transformación urbana.

\begin{abstract}
The theories that support the idea of city are based on a rational consensus to the existence of subjects with the same rights represented by democratic and efficient governments. However, in underdeveloped countries conceptual basis, on which the idea of a city is build, differs from that established in countries with more developed democratic structures and social practices.

Impact of real estate development generated by capitalist production of cities in Latin America has not only accelerated processes of territorial segregation and social conflicts, but they have also exceeded the capacity to plan, finance and manage urban development by local and national governments. Real estate dynamic has affect city meaning and their relations, without been able to build a new city idea that effectively integrates this dynamic and not only reject it.
\end{abstract}

\footnotetext{
${ }^{1}$ Arquitecta, M.Sc., Tecnológico de Monterrey; ${ }^{2}$ Arquitecto, M.Sc., Tecnológico de Monterrey; * Correo de contacto: lorena.sicilia@tec.mx
} 


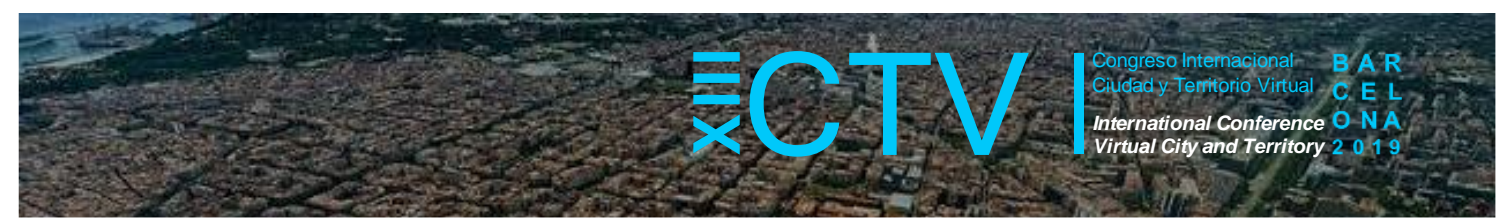

In Mexico City, we find one of the best examples of urban transformation processes in which it is very clear that planning and management models of the city are totally exceeded, but it also presents a great opportunity to rethink models before implementation of a new legal framework: the first Political Constitution of México City. In this study case is explained the existing urban problems and it is presented a methodological proposal for local administration to have better tools to perform a systemic evaluation of urban impacts of real estate development, as well as an example of how they can reduce and compensate for its effects.

This proposal is based on a case -currently under development- carried out by Tecnologico de Monterrey in Mexico City campus. Its objective is that this methodology for evaluation of urban impacts becomes a tool from which urban development planning instruments become quantitative instruments and not only indicative ones, establishing a direct relation between develop and financing, at least at local level.

Palabras Clave: Desarrollo urbano; desarrollo Inmobiliario; impacto urbano; Ciudad de México

Key words: Urban development; Real Estate development; Urban Impact; Mexico City

\section{Introducción}

La ciudad es, sobre toda consideración, un sistema complejo de interrelaciones en constante transformación. En este sentido es necesario cuestionarse y desconfiar de todo determinismo ya que toda la historia y el futuro de las ciudades depende de las representaciones que se hacen sus diversos actores sobre lo que es justo y lo que no lo es, así como de las reacciones de fuerza entre estos actores y de las acciones que resultan de ello. Es por ello que debemos aceptar -y en consecuencia evaluar- que el desarrollo y crecimiento de la ciudad pone en juego poderosos mecanismos que empujan tanto en el sentido de la convergencia como el de la divergencia, algo que hoy día es más patente.

Lo anterior es el resultado -entre muchos otros factores- de que en los últimos años la ciudad ha sido el objeto predilecto del capitalismo global (corporativos de oficinas, viviendas, centros comerciales, hoteles y un largo etcétera.) tal vez como nunca antes en su historia, tal es el caso de la Ciudad de México (CDMX). Esta dinámica de inversión inmobiliaria se ha visto fuertemente rechazada por amplios sectores de la ciudadanía al percibirla como un factor de contrario a su calidad de vida, a su patrimonio, al medio ambiente, a la equidad socio territorial y particularmente a su idea preconcebida de la ciudad en sus distintas escalas.

Asumiendo entonces que la dinámica de la ciudad es sistémica y que no conoce ni reconoce delimitaciones territoriales, que la fuerza y dinamismo del capital inmobiliario es capaz de socavar los instrumentos de ordenamiento y desarrollo de las ciudades, que los grupos sociales y gremios tradicionales pareciera que dejan de ser un factor de cambio real en la ciudad; tenemos entonces que replantear y concebir una nueva idea de ciudad que reconozca su complejidad funcional, las nuevas necesidades sociales y desarrolle los mecanismos y herramientas necesarias para lograrla.

\subsection{Disputa y convergencia por la ciudad}

En el caso de la CDMX el mejor ejemplo de divergencia son los mismos instrumentos de planeación urbana establecidos en la propia ley. En ella se establecen cuatro instrumentos, cada uno de ellos en distinta escala de planeamiento: El Programa General de Desarrollo Urbano (PGDU), los Programas Delegacionales de Desarrollo Urbano (PDDU) (para cada una de las 16 Alcaldías), los Programas Parciales de Desarrollo Urbano (PPDU) (para zonas con 


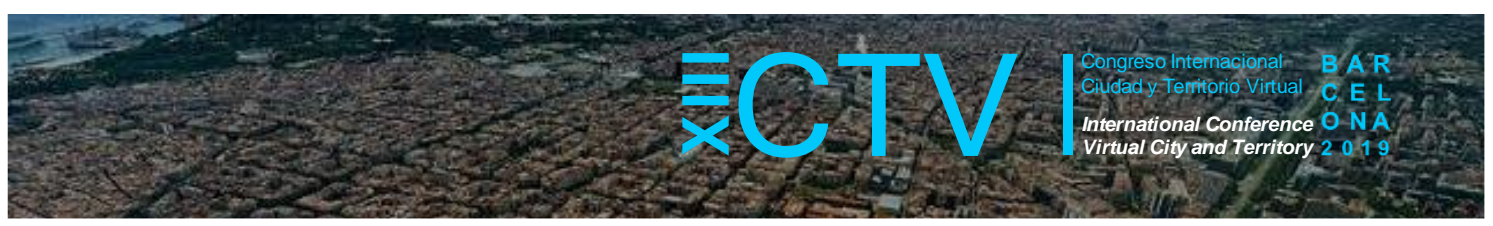

potencial de crecimiento o de valor histórico) y las Áreas de Gestión Estratégica (AGE) (para zonas de regeneración o reconversión).

Prácticamente ninguno de los anteriores ha demostrado ser un instrumento ni de planeación ni de ordenamiento eficiente dado que no reconocen la condición sistémica de la ciudad, la dinámica del capital para transformar los territorios en su exclusivo beneficio, las demandas sociales (particularmente el de la vivienda nueva, la cual se estima en cerca de 85,261 viviendas anuales ${ }^{2}$ lo que no es menor en una ciudad con 2,599,081 viviendas) así como las necesidades en materia de infraestructura, equipamientos y dotación de servicios que el propio plan de desarrollo demandaría. Así mismo ninguno de los instrumentos plantea una idea de ciudad y en consecuencia se encuentran desvinculados de los instrumentos económicos y financieros para alcanzarla.

En la práctica, la mayoría de ellos se han convertido en instrumentos que buscan sin éxito contener la inversión inmobiliaria, mientras que otros han resultado condescendientes a esta. En su conjunto operan como instrumentos de segregación socio económica al fomentar el encarecimiento del suelo y con ello generar un mayor desequilibrio socio territorial.

La CDMX Hoy se cuenta con una gran oportunidad para transformar esta situación con la implementación de la primera Constitución Política de la $\mathrm{CDMX}^{3}$ a partir de la cual se deben reformular las leyes que regulan el desarrollo urbano y medioambiental de la ciudad. En ella se establece por primera vez que "esta Constitución garantiza el derecho a la ciudad a través de instrumentos de planeación, jurídicos, administrativos, financieros, fiscales y de participación ciudadana para hacer efectivas las funciones social, económica, cultural, territorial y ambiental de la ciudad". Sin embargo, aún no se define como se lograrán estos objetivos y es aquí donde se inscribe la oportunidad de plantear soluciones e instrumentos para la generación de recursos de tipo "no tributarios" derivados de la inversión inmobiliaria, así los mecanismos para su gestión en las distintas escalas de planeamiento.

\subsection{La inversión inmobiliaria en la CDMX}

En los últimos 20 años el desarrollo inmobiliario en la CDMX ha tenido un importante crecimiento, la construcción de oficinas, hoteles, centros comerciales y viviendas para segmentos medios y altos han sido los principales protagonistas. Dimensionar este crecimiento no siempre resulta fácil ya que el sector público no difunde datos estadísticos al respecto, por lo que conocer a detalle cuantos metros cuadrados se han construido para cada uso y donde se han construido en el tiempo, es sumamente complejo.

El sector privado es quien en ocasiones da a conocer ciertos datos a partir de los cuales se puede inferir el estado que guarda el desarrollo inmobiliario en la ciudad. Para este caso tomamos la información difundida por la Asociación Nacional de Desarrolladores Inmobiliarios (ADI), la que si bien no agrupa a la totalidad de quienes invierten en el sector, si agrupa a la mayoría y a la más importantes del sector.

\footnotetext{
${ }^{2}$ Sociedad Hipotecaria Federal (SHF) Demanda de financiamiento de vivienda 2019. México 2019.

${ }^{3}$ Constitución Política de la Ciudad de México.
} 


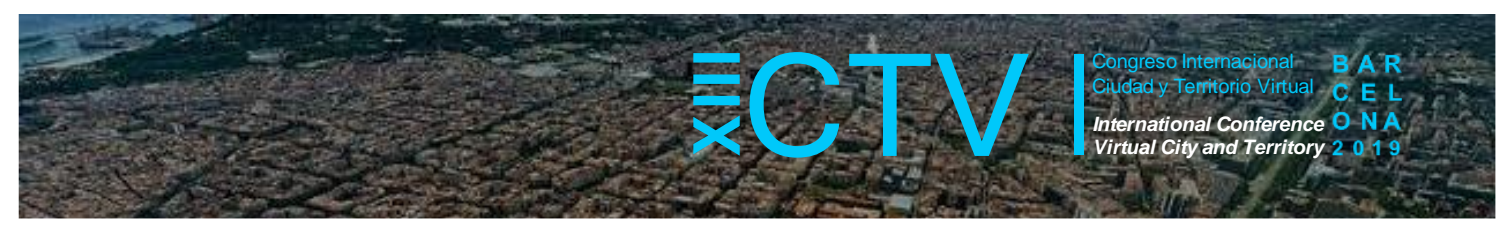

Según estos datos, en tan solo tres años (2013-2016) los miembros de la ADI construyeron $14,340,729 \mathrm{~m}^{2}$ en la Ciudad de México. Esto representa una inversión estimada de $\$ 8,394,179,430$ USD lo que equivale a $\$ 735$ USD por $\mathrm{m}^{2}$ promedio. Esta inversión hasta cierto punto habría que celebrarla por las implicaciones económicas que por si conlleva, sin embargo, y como veremos a continuación, ha sido generadora de importantes externalidades negativas a partir de las cuales el rechazo ciudadano y los procesos de divergencia y convergencia de los que hablamos anteriormente se han recrudecido en la CDMX.

La inversión inmobiliaria se ha desarrollado de forma muy desigual en el territorio, el periodo analizado es muestra de ello. El $53 \%$ de lo edificado se concentró en una sola Alcaldía y otro $25 \%$ en tan solo otras dos, de hecho, en otras cinco Alcaldías no hubo inversión alguna y por ello no aparecen en los gráficos.

Figuras 1 y 2. metros cuadrados construidos por alcaldía en la CDMX (2013-2016)

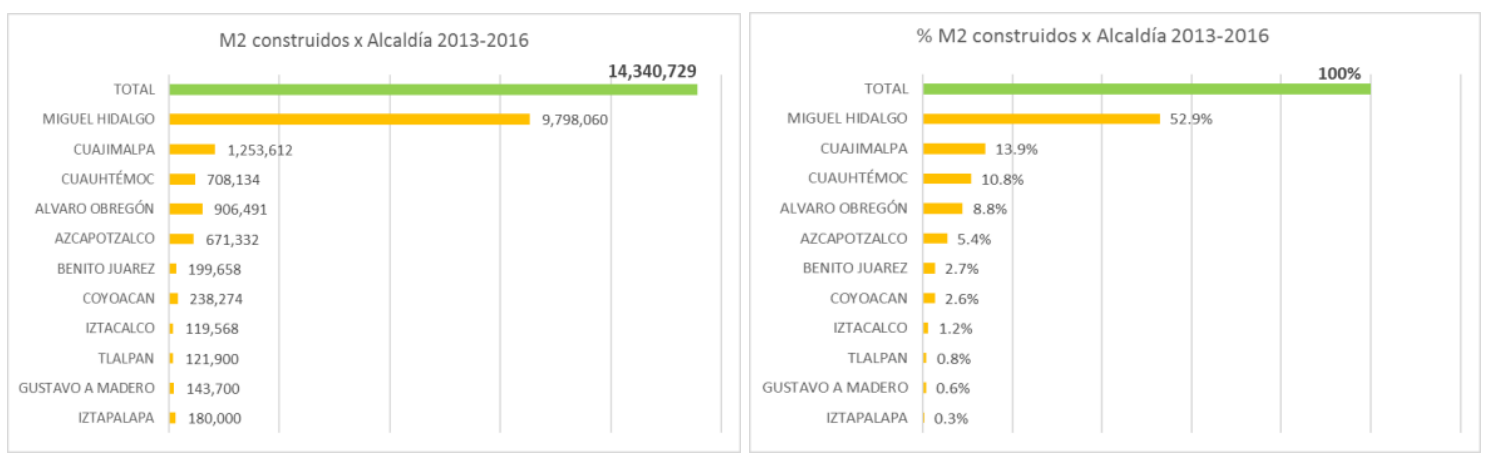

Fuente: elaboración propia con información de ADI

Sin embargo, la problemática no se queda en la concentración territorial, sino también en los impactos que la construcción de todos estos metros cuadrados genera en la ciudad y la forma en que deben compensarse, lo que necesariamente requiere de recursos económicos. En este sentido es necesario evaluar los recursos que la ciudad recibe por permitir la construcción. El código fiscal solo reconoce 11 rubros de cobro para la construcción (licencias y permisos), los que en promedio representan el 4.5 del costo directo de la construcción. En este ejemplo lo recaudado fue de tan solo $\$ 381,767,280$ USD, de ellos únicamente el $9 \%$ son recaudados por las Alcaldías y el restante $91 \%$ por la CDMX.

En ese reconocimiento la actual Ley de Desarrollo Urbano prevé en su reglamento un mecanismo a partir del cual es posible evaluar los impactos urbanos de algunos proyectos y la forma en que pueden compensar o mitigar para que se integren de manera eficiente en su contexto urbano.

\subsection{La evaluación del impacto urbano en la Ciudad de México}

La Ley de Desarrollo Urbano y su reglamento establece que los proyectos de construcción que superen los $5,000 \mathrm{~m}^{2}$ de construcción en cualquier uso y de $10,000 \mathrm{~m}^{2}$ para proyectos de vivienda social, son objeto de un Estudio de Impacto Urbano (EIU), esto con la finalidad de "evaluar y dictaminar las posibles influencias o alteraciones causadas al entorno urbano o urbano ambiental por algún proyecto público o privado en el área donde se pretenda realizar, 


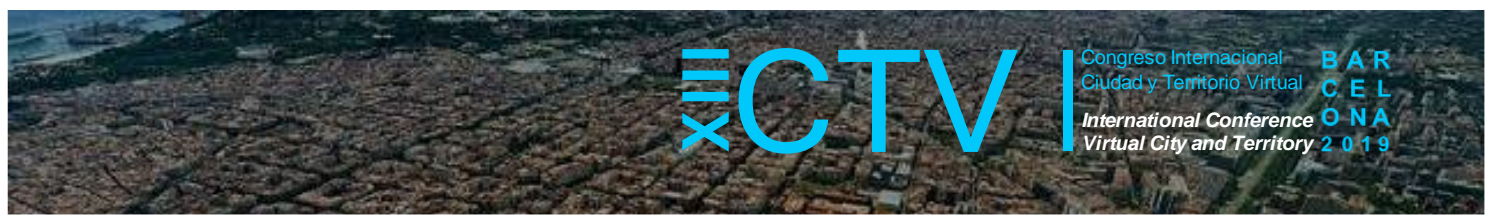

con el fin de establecer las medidas adecuadas para la prevención, integración y/o compensación"4

Así mismo establece que dicha evaluación estará a cargo de un Perito en Desarrollo Urbano (PDU) -en teoría un auxiliar de la administración pública- quien deberá realizar una "Propuesta de Medidas de Integración Urbana y su Programa de Ejecución" la cual será evaluada por las distintas dependencias gubernamentales involucradas. Sin embargo, no existe una metodología puntual sobre la determinación o definición de lo que sería el "entorno inmediato al proyecto", los alcances que debe integrar el diagnóstico, las fuentes de información y en consecuencia los alcances de la propuesta, las acciones a realizar ni los criterios sobre los cuales esta será evaluada.

Si los proyectos inmobiliarios que utilizamos como ejemplo hubieran sido objeto de EIU -dado que ciertamente la mayoría de cada uno de ellos fue mayor a 5,000 $\mathrm{m}^{2}$ - y como parte de ello hubiera destinado en promedio el $4 \%$ de la inversión -un número que ha sido demostrado empíricamente en diversos proyectos- para mitigar y mejorar su contexto urbano, el monto destinado a ello hubiera sido de al menos $\$ 419,708,972$ USD, sin embargo en la práctica, el monto destinado para Medidas de integración Urbana (MIU) es cercano al $0.7 \%$ lo que equivaldría a tan solo $\$ 2,937,962$ USD.

Lo anterior es consecuencia no solo de la falta de una metodología clara de diagnóstico y evaluación, sino también de al menos tres factores calve: el primero es que los proyectos objeto de EIU se definen por un metraje de construcción determinado $\left(5,000 \mathrm{~m}^{2}\right) \sin$ considerar las características del contexto urbano, tampoco se determinan en función del tipo e intensidad de uso a los que se destinan; en segundo lugar, la administración pública no cuenta con información sobre las capacidades reales de la infraestructura y servicios instalada de cada una de las zonas donde puedan implementarse los proyectos -por lo que las evaluaciones resultan subjetivas- $y$ finalmente por el hecho de que los servicios del PDU son pagados por el desarrollador inmobiliario, generando así un claro conflicto de intereses.

Para plantear una solución en términos metodológicos sobre esta situación y como parte que desarrolla el Tec de Monterrey que por sus características es objeto de al menos cinco EIU, se planteó una propuesta metodológica para la realización de los EIU, así como para las MIU y su respectivo diseño y evaluación económica.

\section{Caso de estudio}

El día 19 de septiembre del 2017 en la CDMX ocurrió uno de los terremotos más devastadores de su historia contemporánea, el sismo de magnitud 7.1 dejó 300 personas fallecidas, al menos 40 edificios colapsados, reportes de daños graves en al menos 500 inmuebles. Entre los edificios afectados están las instalaciones del Campus Ciudad de México (CCM) del Tecnológico de Monterrey (TEC) en los que resultaron con daños irreparables 75,000 $\mathrm{m}^{2}$ de los $90,000 \mathrm{~m}^{2}$ con los que se contaba y en los cuales se congregaba una comunidad universitaria de 9,000 personas entre alumnos, docentes y colaboradores.

\footnotetext{
${ }^{4}$ Reglamento de la Ley de Desarrollo Urbano del Distrito Federal, articulo 85, pág.42. Ciudad de México 2018.
} 


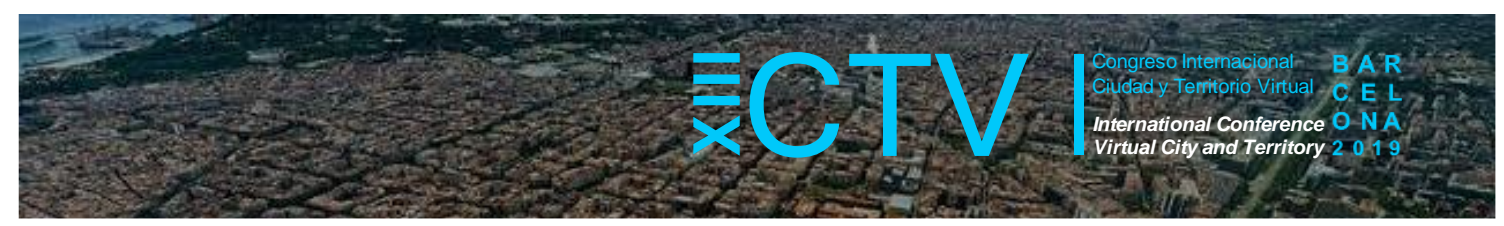

El proceso de reconstrucción del Campus ha enfrentado diversos retos entre los que destacan la no suspensión de sus actividades académicas en instalaciones temporales, la demolición de los edificios existentes, el diseño y construcción de nuevas instalaciones. También se adquirieron predios contiguos al CCM con una superficie de $133,200 \mathrm{~m}^{2}$ que sumados a los pre existentes dan un total de $339,131 \mathrm{~m}^{2}$.

Figura 3. Mapa de los predios del Tec de Monterrey. En verde se muestran los predios que fueron adquiridos posterior al sismo de 2017

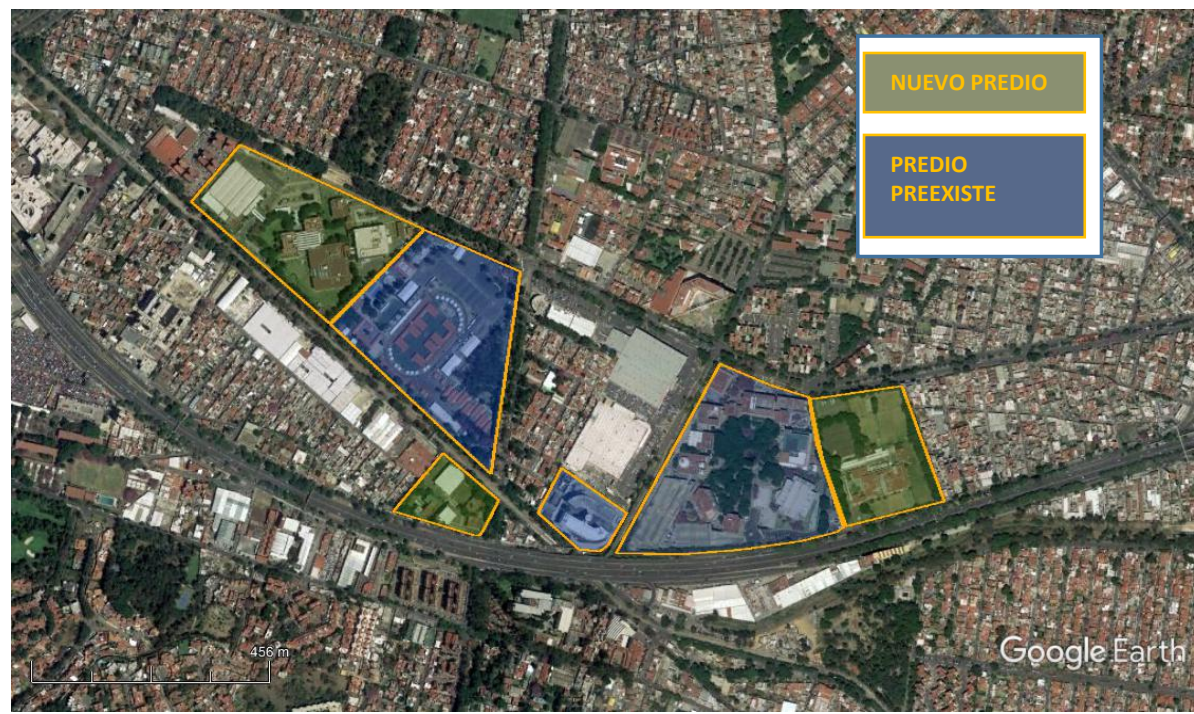

Fuente: Google Earth / elaboración propia

Estas nuevas condiciones nos permitirán ampliar las instalaciones en hasta 608,000 m2 en un periodo de 6 años. Estas nuevas condiciones de crecimiento y desarrollo nos obligan a replantear la relación del Campus con su entorno urbano y social, así como reconsiderar el impacto urbano del Campus y sus respectivas externalidades.

En este marco, el Tecnológico de Monterrey se planteó el desarrollo de una metodología que permitiera definir e implementar las Medidas de Integración Urbana no solo para este caso de estudio, sino para cualquier tipo de proyecto que por su escala e impacto urbano se desarrolle en la CDMX.

La metodología parte de la definición y análisis exhaustivo de la condición socio territorial de un polígono de estudio que en este caso comprendió una superficie de $322 \mathrm{Ha}$. compuesto por 15 barrios que integran 235 manzanas (bloques) subdividíos en 5,309 lotes (predios) con 27,927 habitantes y una población flotante de 41,300 habitantes al día.

El polígono se caracteriza por integrar diversos sectores socioeconómicos, una condición sociodemográfica en el que prevalece el despoblamiento y el envejecimiento poblacional, así como por su alta concentración de equipamientos ente los que destacan: 32 equipamientos de servicio público, 35 equipamientos de servicio privados. Del total de equipamientos 30 son de educación, 8 de abasto y comercio, 9 de deporte y recreación, 9 de asistencia social y salud y 11 de administración urbana y servicios. 


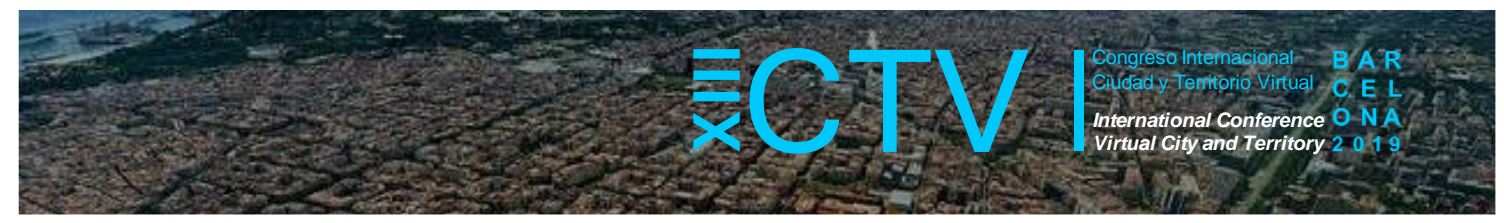

Figura 4. Mapa de localización del polígono de estudio respecto a la ciudad

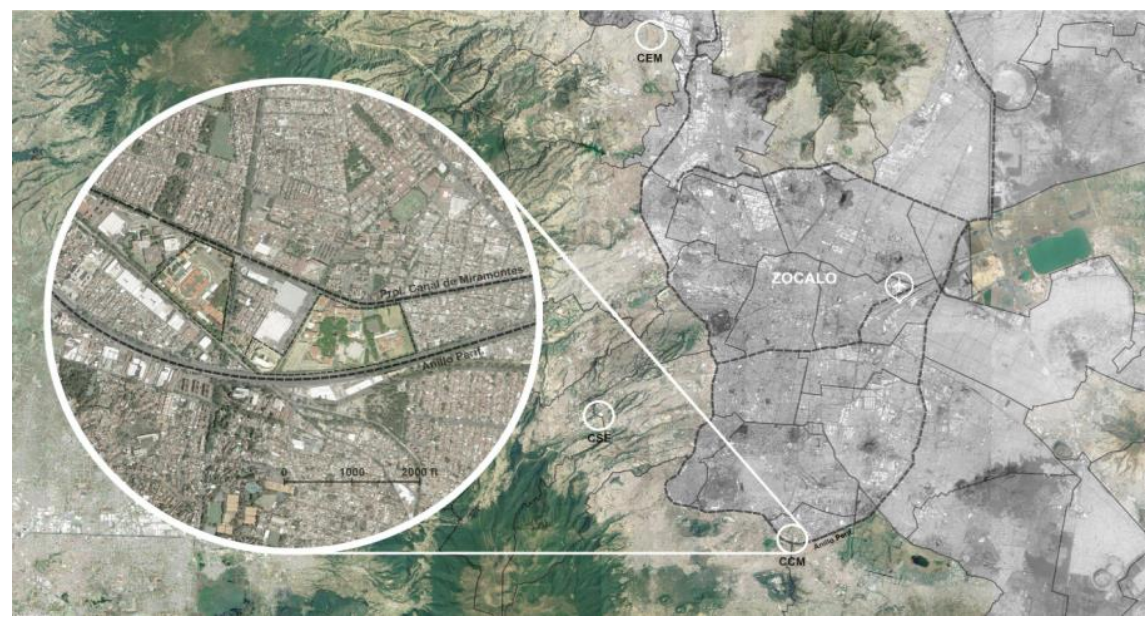

Fuente: Elaboración propia

Figura 5. Densidad. Habitantes por manzana en el polígono de estudio

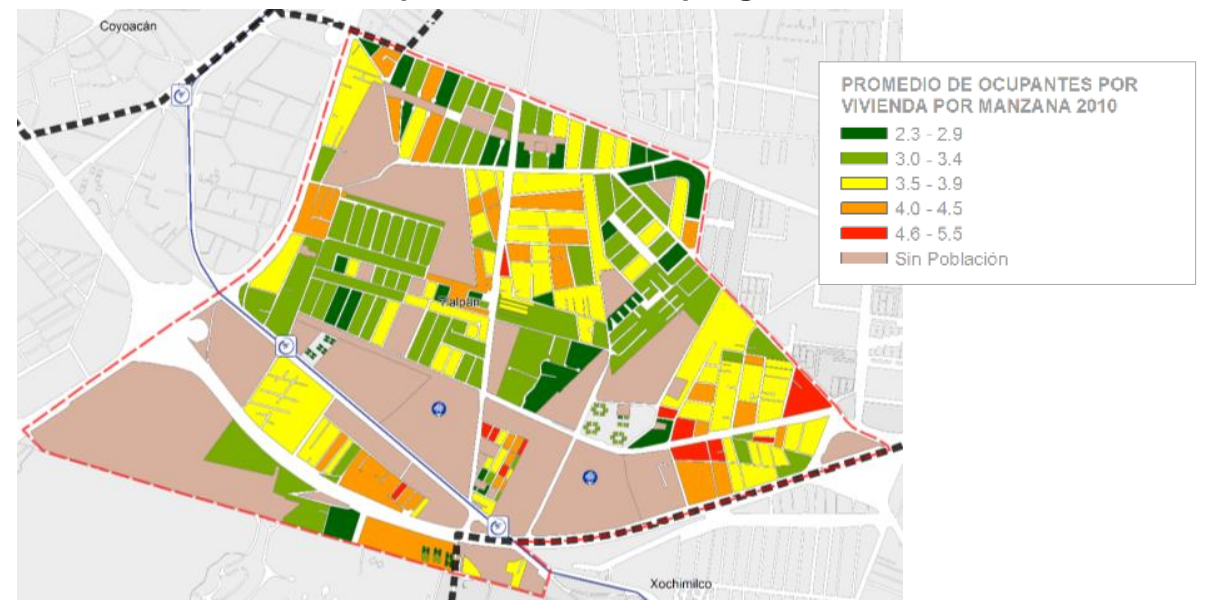

Fuente: World Resources Institute, WRI

Figura 6. Mapa de equipamientos en el polígono de estudio

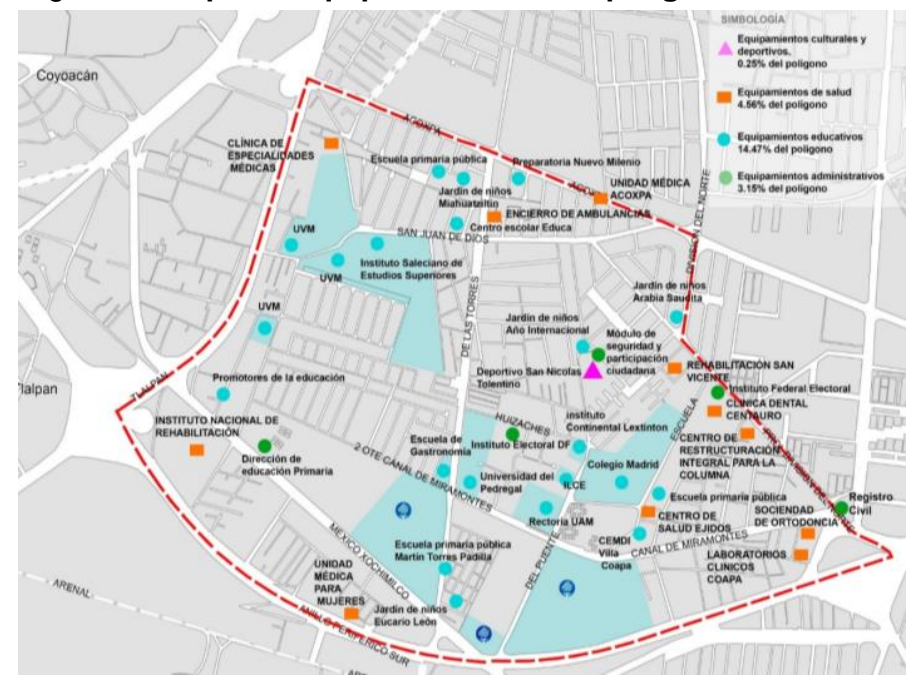

Fuente: elaboración propia 


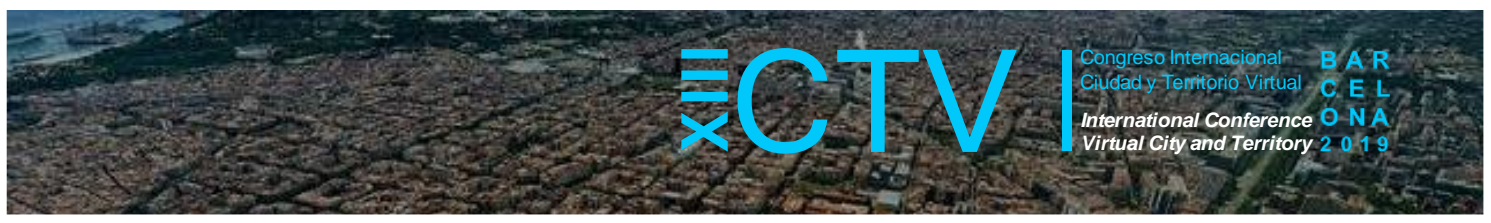

\subsection{Definición Metodológica}

La importancia de una metodología para definir las medidas de integración en cualquier zona de la ciudad, se convierte en una necesidad primordial que brinda la oportunidad de conocer la ciudad a fondo, permite a su vez el financiamiento para la regeneración y mejoramiento del espacio abierto, así como unifica y sistematiza la manera en que los PDU integran los Dictámenes de Impacto Urbano. Para poder sistematizar la información obtenida y traducirla a proyectos de regeneración de infraestructura y espacio público, determinamos los pasos generales para llevar a cabo este proceso:

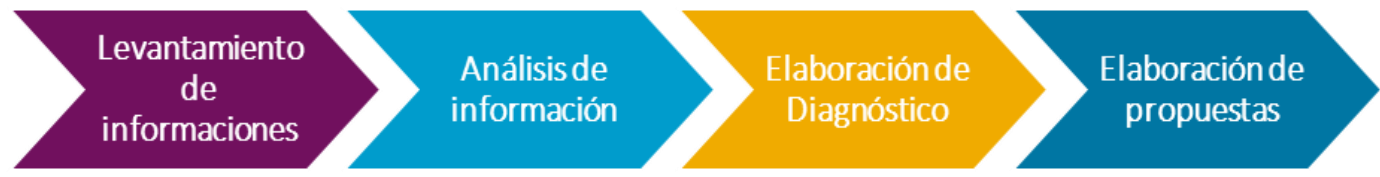

En lo particular para poder establecer este análisis sistémico se definieron los rubros que se muestran en la figura 7 :

Figura 7. Metodología para definición de las medidas de integración a partir de un polígono DEFINICIÓN DEL POLÍGONO

\begin{tabular}{|c|c|c|c|c|}
\hline Análisis Normativo & $\begin{array}{c}\text { Caracterización } \\
\text { Urbana }\end{array}$ & $\begin{array}{c}\text { Caracterización } \\
\text { Social }\end{array}$ & $\begin{array}{c}\text { Caracterización } \\
\text { Ambiental }\end{array}$ & $\begin{array}{l}\text { Caracterización } \\
\text { Económica }\end{array}$ \\
\hline $\begin{array}{l}=\text { PGDU } \\
" \text { PDDU } \\
" \text { PPDU } \\
=\text { AGE }\end{array}$ & $\begin{array}{l}\text { Movilidad } \\
\text { Vivienda } \\
\text { Espacio Público } \\
\text { Equipamiento } \\
\text { Consumos y residuos } \\
\text { Potencial constructivo }\end{array}$ & $\begin{array}{l}\text { Grupos de edad } \\
: \text { PEA/ PO } \\
: \text { Población residente } \\
: \text { Población visitante } \\
\text { Indice de marginación } \\
\text { Violencia }\end{array}$ & $\begin{array}{l}\text { Corredores ambientales } \\
\text { " Cuerpos de agua } \\
\text { Vegetación } \\
\text { Riesgos } \\
\text { Espacio abierto }\end{array}$ & $\begin{array}{l}\text { Unidades económicas } \\
\text { básicas } \\
\text { Valor del suelo } \\
\text { Valor de vivienda } \\
\text { Recaudación de } \\
\text { impuestos }\end{array}$ \\
\hline
\end{tabular}

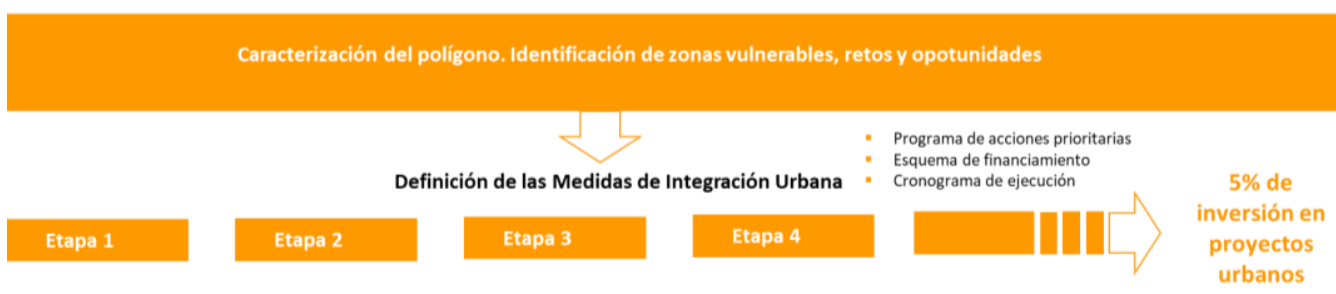

Fuente: elaboración propia

\subsection{Definición del polígono y su caracterización}

En primer lugar, es necesario definir el polígono de estudio conformado por el entorno inmediato al predio o predios donde se prevé el desarrollo inmobiliario y un área de influencia o contexto territorial de mayor alcance que albergará los barrios circundantes o proyectos en puerta. En lo que al análisis normativo respecta, resulta fundamental conocer el marco normativo del desarrollo urbano para la zona delimitada, pues los usos y potenciales constructivos pueden variar de manera significativa de un bloque a otro. Este análisis permite identificar el potencial constructivo de todo el polígono, que junto al tamaño de los predios (solares) y su localización nos permite identificar el desarrollo de proyectos en el corto y mediano plazo. En lo concerniente a la estructura o caracterización urbana, nos referimos al reconocimiento de las condiciones urbanas generales del polígono en términos de usos y aprovechamientos del suelo y de su potencial de desarrollo bajo las normas urbanísticas actuales. Asimismo, estudia la oferta de equipamientos y servicios actuales de la zona, su 


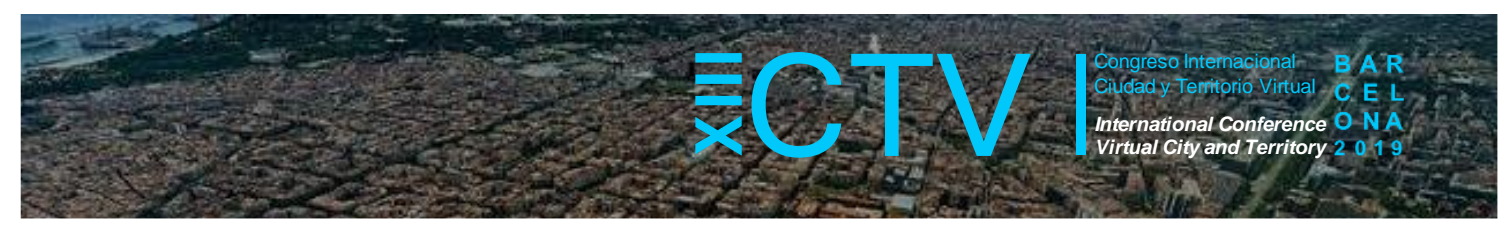

accesibilidad a nivel local y con respecto al resto de la ciudad, identifica la tipología de vivienda (unifamiliar o plurifamiliar y su condición propietaria (propia o en alquiler) que la compone, el consumo promedio de recursos hídricos y energéticos, así como las descargas sanitarias y residuos sólidos que se producen.

A partir de los resultados del coeficiente de ocupación de la superficie (COS) y coeficiente de utilización de la superficie (CUS), es posible calcular el potencial de aprovechamiento del suelo para cada una de las manzanas del polígono, entendido éste como el total de metros cuadrados que es posible construir, tomando como referencia el uso de suelo programado, esto para hacer un análisis de la capacidad de crecimiento, densificación que pueda presentar en un escenario de corto y mediano plazo.

Hablando de capital social o caracterización social, es primordial comprender la composición poblacional del polígono; las dinámicas sociales que se generan en el mismo y la manera en que los miembros de la comunidad interactúan entre sí. Sabemos que la cohesión social de una comunidad puede permanecer estable en el tiempo, pero también puede erosionarse 0 fortalecerse, en ese sentido, cualquier medición que se haga de la misma será necesariamente una fotografía acotada a un momento determinado en el tiempo (México Evalúa, Fundación Hogares; 2015). Sin embargo, nos permite conocer en ese momento la disponibilidad al cambio y transformación urbana, las necesidades de la comunidad de primera mano, convirtiéndose en aliados para la ejecución de los proyectos.

En materia medioambiental, el conocimiento del ecosistema local se vuelve relevante para promover el fortalecimiento de procesos biológicos del sitio; las dinámicas hídricas que en ocasiones pueden ser factor de riesgo en la zona de estudio como es el caso del polígono en cuestión. Finalmente, analizar con la base estadística existente en los censos económicos y evaluación de campo a nivel barrial para identificar las características de los mercados locales y la vocación económica de la zona. A partir de la generación de indicadores sobre el tipo de actividad económica del polígono (comercios, servicios, actividades productivas, empleos, aportación económica, etc.) para definir la vitalidad económica y su potencial de crecimiento en función de la población residente y visitantes. Adicionalmente, conocer el costo de impuesto prediales y la recaudación fiscal generada, así como el valor y dinámica del mercado de la vivienda, oficinas y comercios. Integrar estos datos permite hacer cruces de información para establecer escenarios sobre el potencial de transformación que tiene esa zona a futuro y los ingresos tributarios que genera a la ciudad.

Con esta integración es posible identificar el "peso específico" de cada zona del polígono y de cada nuevo proyecto en relación a la capacidad de la infraestructura y servicios existente. Si la capacidad instalada es suficiente para sostener los nuevos proyectos el monto de inversión para ejecutar MIU será menor, en caso contrario las MIU deberán cubrir los costos directos que ello implique, atendiendo de manera prioritaria el tipo de impacto causado (movilidad, transito, medio ambiente, servicios hídricos, descargas, residuos, etc.) Con ello se determina si el proyecto en cuestión es factible o bajo que condicionantes puede ser realizado.

Adicionalmente se realizaron entrevistas y talleres de participación social en los barrios que conforman el polígono de estudio cuyo objetivo era el de identificar problemáticas comunes relacionadas a la infraestructura y servicios, así como la percepción social sobre temas de inseguridad y responsabilidad sobre quien debería solucionar estas problemáticas. De ello se 


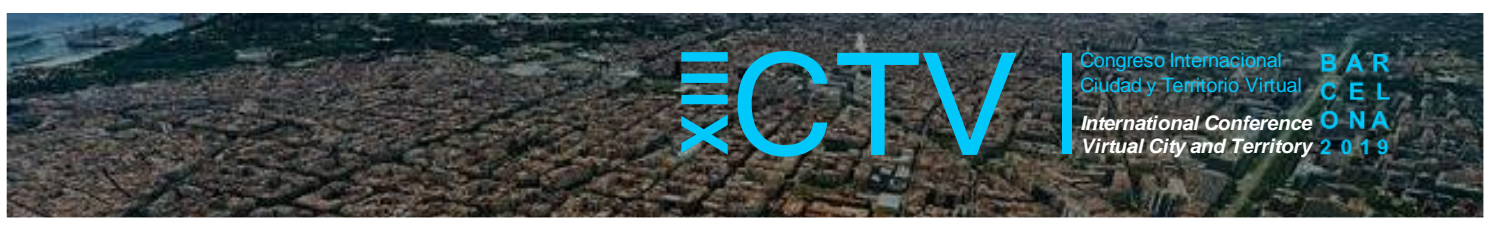

obtuvo un plano síntesis de problemáticas en el polígono a partir del cual se identificaron 21 puntos problemáticos del que se desprendieron 15 proyectos de infraestructura urbana que deberán ser atendidos como parte de las MIU del proyecto.

En este caso de estudio, los principales impactos detectados se relacionan con temas de movilidad y accesibilidad (tránsito vehicular, accesibilidad al transporte público e infraestructura peatonal) dado el número de viajes que genera la comunidad del Campus; así como el uso intensivo de la infraestructura hídrica existente. Para atender estos impactos se desarrollaron proyectos de diseño e ingeniería (en los 15 proyectos identificados) a partir de los cuales se evaluaron los costos de ejecución.

Coincidentemente al análisis empírico de otros proyectos en la ciudad, el monto de inversión proyectado es equivalente al $4.8 \%$ del costo directo de construcción para el proyecto. Este monto podría parecer elevado y se podría pensar que sería un factor que detuviera la inversión, sin embargo, en la CDMX los proyectos inmobiliarios tienen una Tasa Interna de Retorno (TIIR) generalmente superior al $20 \%$ de la inversión, por lo que en un análisis de costo beneficio este monto no sería un factor que limite la inversión. Además, esta inversión en infraestructura se transforma en un factor adicional para la generación de plusvalías, por lo cual podrá ser recuperado en el tiempo.

\section{Figura 8. Mapa síntesis de las principales problemáticas identificadas en el polígono en} materia de seguridad, movilidad, infraestructura hídrica y déficit de equipamiento

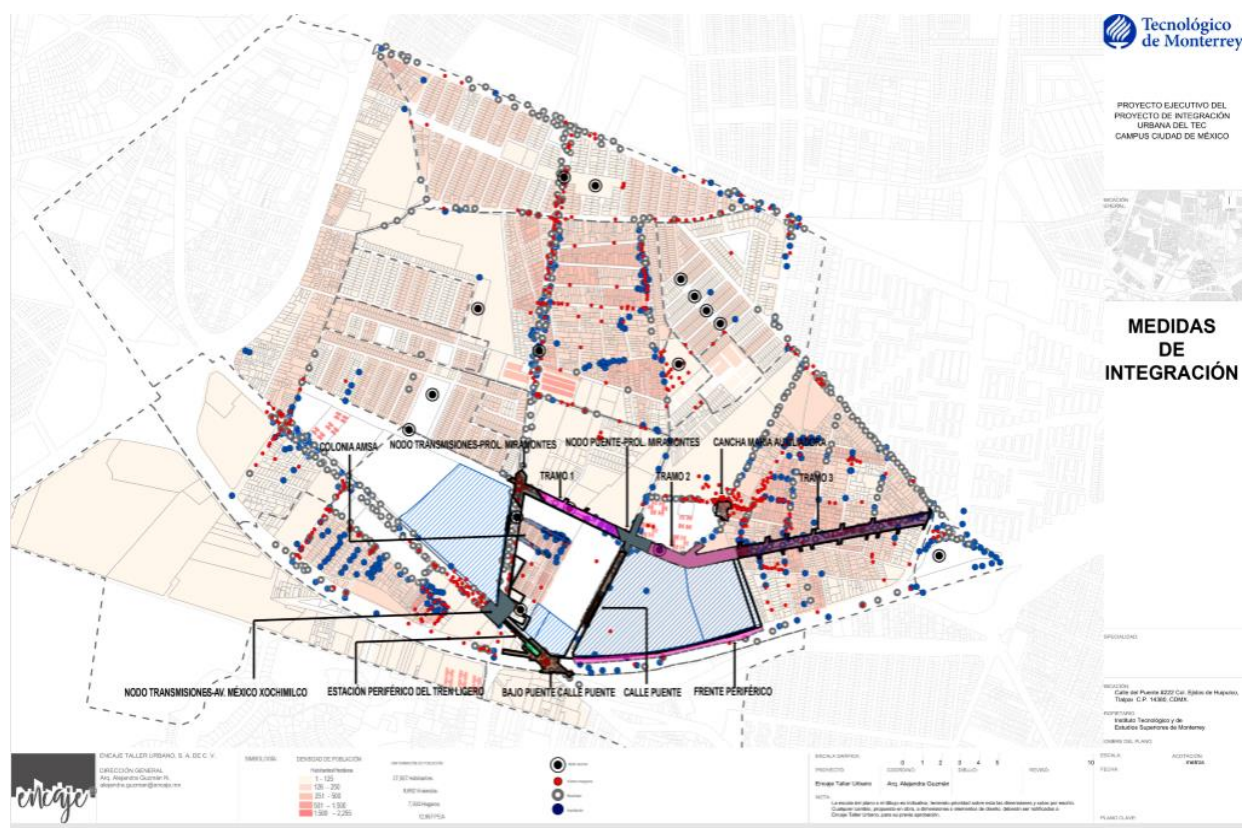

Fuente: elaboración propia

Es evidente que la solución total de las problemáticas identificadas no puede ser resuelta mediante las MIU de un solo proyecto, pero la integración de varios proyectos puede generar recursos suficientes para atender las principales problemáticas. Para que ello sea efectivo es necesario diseñar una estructura operativa y de gestión que sea responsable tanto de evaluar a nivel barrial las problemáticas como de administrar los "ingresos no tributarios" derivados de las MIU y coordinar las acciones y obras que deban realizarse. 


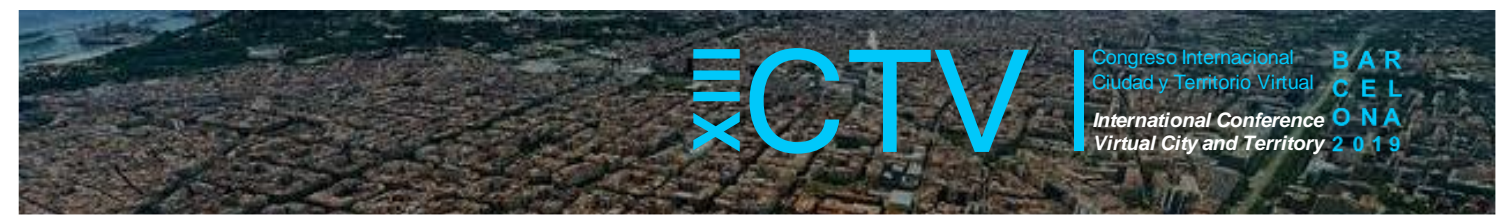

Figura 9. Mapa síntesis de los proyectos de Medidas de Integración Urbana

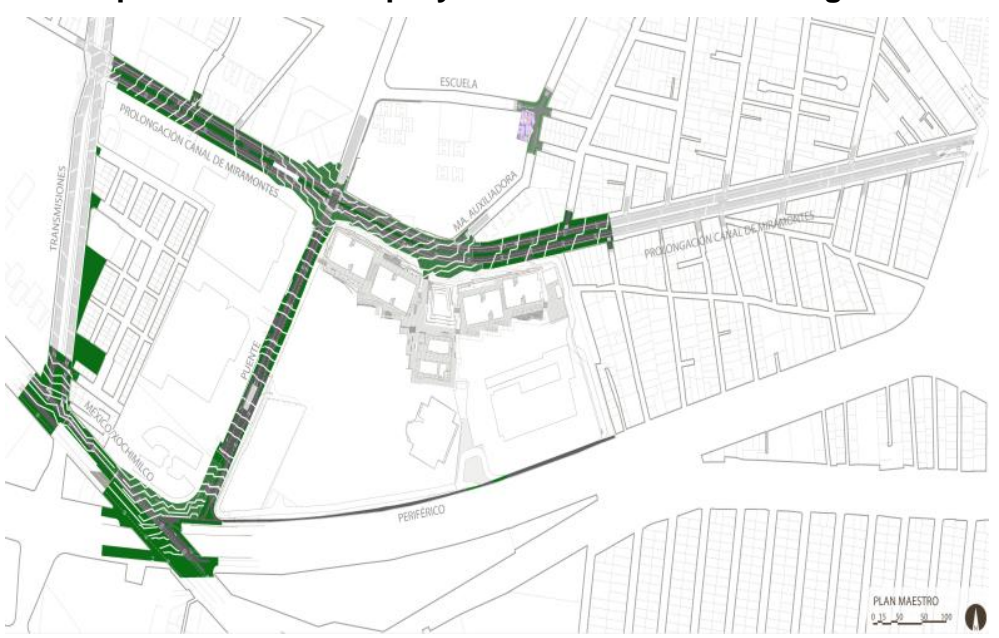

Fuente: elaboración propia

Figuras 10 y 11. Imagen objetivo proyecto Medidas de Integración Urbana. Cancha María Auxiliadora
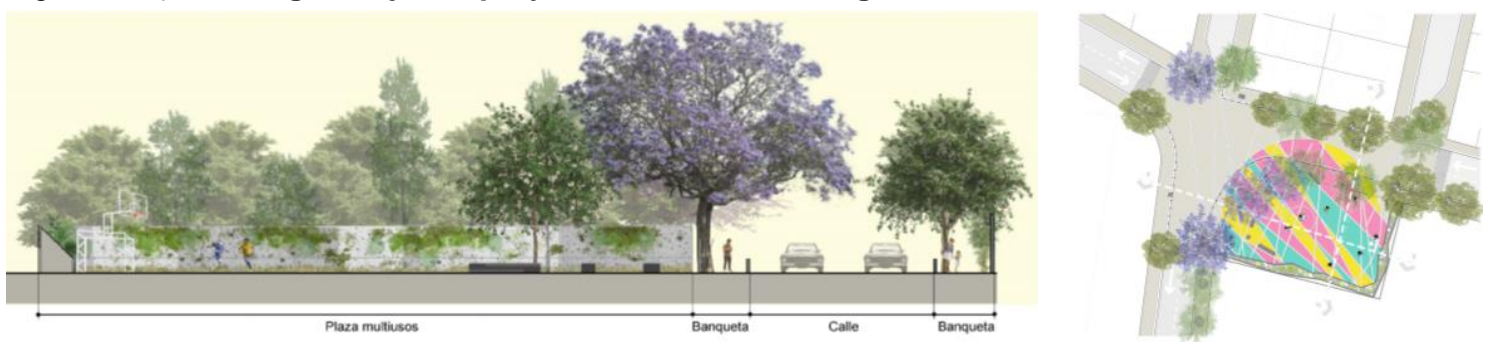

Fuente: elaboración propia

Figura12. Imagen objetivo proyecto Medidas de Integración Urbana. Bajo Puente Anillo Periférico

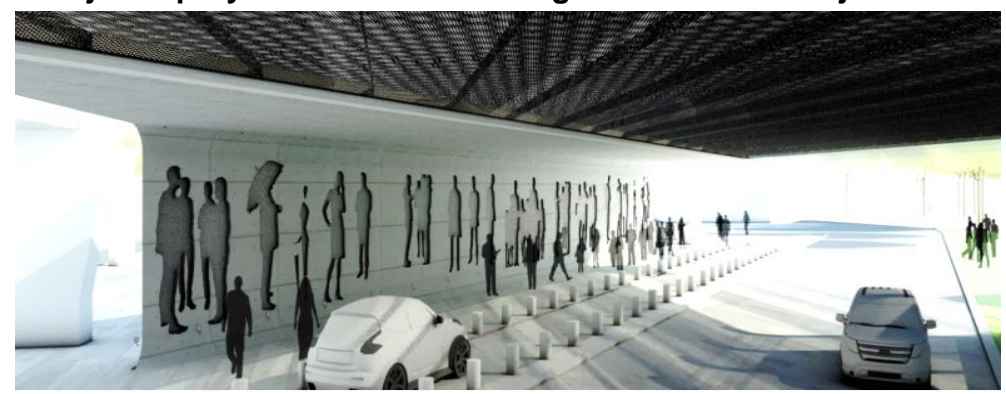

Fuente: elaboración propia

Figura 13. Imagen objetivo proyecto Medidas de Integración Urbana. Sección calle Puente

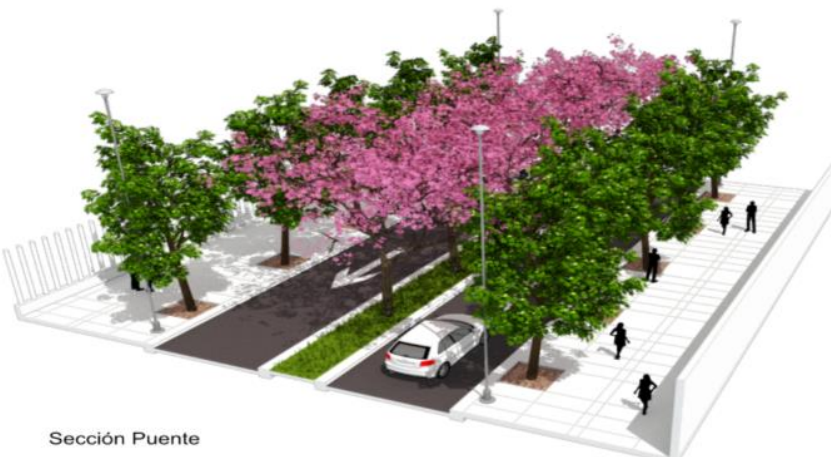

Fuente: elaboración propia 


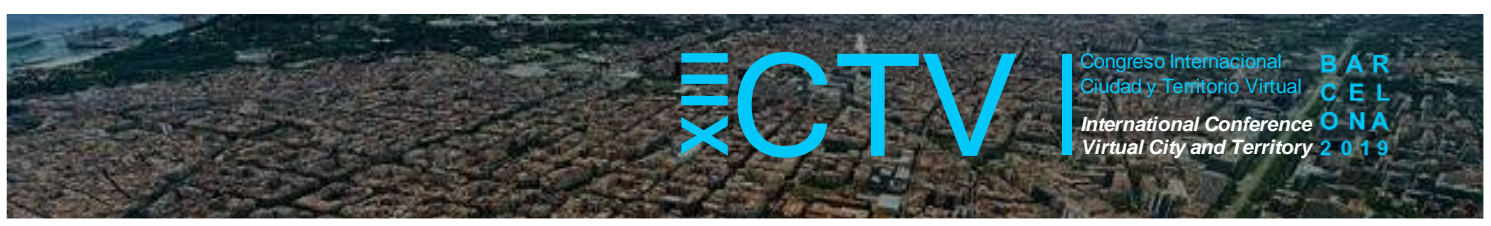

\section{Conclusiones}

Una adecuada evaluación de los impactos urbanos de los proyectos inmobiliarios es tan solo el primer paso para compensar -al menos en términos de escala barrial- las externalidades que estos generan, así como para reducir el conflicto social y territorial que se deriva. Junto con ello se deben integrar otros mecanismos para la recuperación de plusvalías generadas por la normatividad urbana y los procesos de especulación del suelo asociados a las zonas de desarrollo inmobiliario.

Al mismo tiempo se deben diseñar mecanismos de operación y gestión que permitan identificar no solo los rezagos que en materia de infraestructura y servicios presenta la ciudad, sino también proyectar los requerimientos futuros que permitan dar soporte a la dinámica de crecimiento que demanda la ciudad, Ello debe estar acompañado de instrumentos que permitan dar transparencia sobre el origen y destino de estos recursos para con ello generar procesos de participación social efectivos.

La formulación y diseño de las leyes segundarias de la nueva constitución de la CDMX es la oportunidad para idear e implementar nuevas soluciones que permitan disminuir el impacto de la producción capitalista en la ciudad, así como para crear una nueva idea de ciudad en sus distintas escalas. Con este ejemplo buscamos demostrar que ello es posible en la práctica si se logran consensos e incentivos entre los distintos sectores que inciden de manera directa en la ciudad, los que en el caso de la CDMX tienen igual o más efectividad que las leyes y reglamentos que determinan el desarrollo de la ciudad.

Agradecimientos: Nuestro más sincero agradecimiento al Tecnológico de Monterrey por la oportunidad de desarrollar esta iniciativa y de manera especial al Dir. de la Dirección de Urbanismo Ciudadano de esta universidad Jose Antonio Torre Medina y al Vicepresidente de la Región CDMX Rashid Abella.

Contribuciones de los autores: ambos autores han desarrollado de manera conjunta el presente documento en su totalidad.

Conflicto de Intereses: No existen conflicto de intereses en el desarrollo y presentación del presente documento.

\section{Bibliografía}

Constitución Política de la Ciudad de México. 2017. Ciudad de México. Recuperado de http://www.infodf.org.mx/documentospdf/constitucion cdmx/Constitucion \%20Politica CDMX.pdf

DENUE, Directorio Estadístico Nacional de Unidades Económicas. Recuperado de https://www.inegi.org.mx/app/mapa/denue/

Reglamento de la Ley de Desarrollo Urbano del Distrito Federal, de 15 de marzo del 2018, publicado en Gaceta Oficial. Artículo 85, pág.42. Ciudad de México. Recuperado de https://www.seduvi.cdmx.gob.mx/storage/app/uploads/public/5c8/1b4/45c/5c81b445c769f32383 8382.pdf 